\title{
The Pattern Speed of NGC 936 by the Tremaine-Weinberg Method
}

\author{
Konrad Kuijken \\ Kapteyn Institute, PO Box 800, 9700 AV Groningen, Netherlands
}

Michael R. Merrifield

Dept. of Physics, Southampton University, SO17 1BJ, UK

\begin{abstract}
We describe a kinematic measurement of the pattern speed in the classic SB0 galaxy NGC 936. While the practicalities of this method remain difficult, allowance for non-Gaussian velocity distributions enables more reliable measurements than hitherto possible. The bar of NGC 936 appears to end just inside its corotation radius.
\end{abstract}

\section{Introduction: The Tremaine-Weinberg Method}

As in clear from many of the papers in this volume, the pattern speed $\Omega_{p}$ of a bar is a fundamentally important parameter for understanding its structure.

Tremaine \& Weinberg (1984) have devised a method for measuring $\Omega_{p}$ of any flat disk galaxy in a steady state in some rotating coordinate frame. The theoretical basis for the method is the continuity equation (no further dynamical information is required), from which they derive the following relation between the mean velocity $v(x, y)$ and luminosity density $l(x, y)$ in the disk plane:

$$
\Omega_{p}=\left.\frac{\int_{-\infty}^{\infty} d x\left(v_{y}-v_{\mathrm{sys}}\right) l}{\int_{-\infty}^{\infty} d x x l}\right|_{y=\text { const }}=\left.\frac{\text { flux-weighted mean }\left(v_{y}-v_{\mathrm{sys}}\right)}{\text { flux-weighted mean } x}\right|_{y=\mathrm{const}} .
$$

If the $x$ axis is aligned with the intersection between the sky and galaxy planes, then $\left(v_{y}-v_{\text {sys }}\right)$ becomes $\left(v_{r}-v_{\text {sys }}\right) / \sin i$, the radial velocity w.r.t. the systemic velocity $v_{\text {sys }}$ divided by the sine of the inclination of the galaxy, and all quantities in the equation are observable with long-slit spectroscopy. Single long-slit exposures parallel to, but offset from, the $x$-axis yield a value for $\Omega_{p}$, and several such exposures can be used to test the consistency of the result, and to allow for uncertainties in $v_{\text {sys }}$.

In practice the TW method has proved difficult to apply. Most barred galaxies in fact do not appear to be in a sufficiently steady state, or their stellar light does not obey the continuity equation (star formation, which acts as a hard to calibrate source term, spoils continuity). Furthermore, even for the few classic regular, gas-poor, early-type spiral galaxies the integrals are difficult to determine with sufficient precision since the non-axisymmetry signatures are fairly weak. 


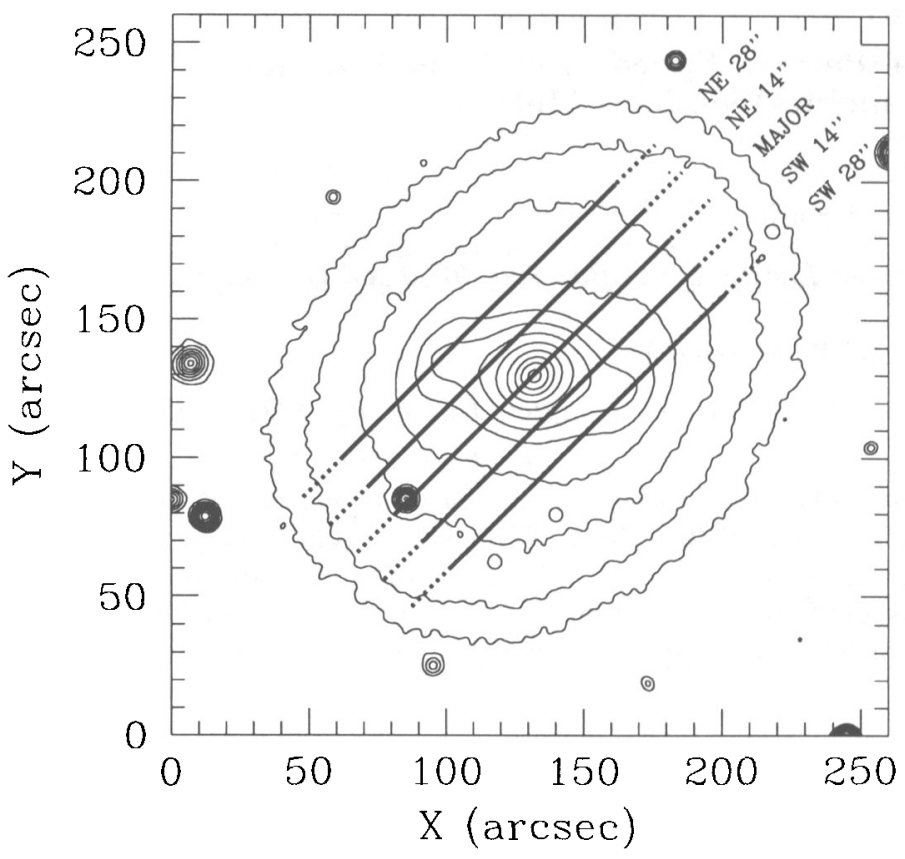

Figure 1. CCD isophotes for NGC 936, and the slit positions observed.

\section{Application to NGC 936}

Here we present the results of a recent analysis of the large SB0 barred galaxy NGC 936. Further details of the work can be found in Merrifield \& Kuijken (1995). Unlike previous work using this method (e.g. Kent 1987, who studied NGC 936 with inconclusive results), we have performed the integrals in 'photon space': we simply co-add all spectra from a single long-slit exposure, and analyze the absorption-line kinematics allowing for asymmetric features in the velocity distribution which might (and do) affect the determination of a mean velocity along the slit.

A CCD image of NGC 936 is presented in Figure 1. At large radii, the isophotes tend to similar ellipses, suggesting that the outer parts of the galaxy are circular. We have therefore taken the major axis of the outer isophotes (PA $45^{\circ}$ ) as the $x$ axis, and obtained five long-slit spectra parallel to this axis with the Multiple Mirror Telescope Red Channel Spectrograph.

Results are shown in Figure 2. Clearly, the signal sought is a small fraction of the full width of the velocity distributions, and therefore good data and careful analysis are required to obtain reliable results. The determination of the pattern speed is then straightforward: a regression of the mean velocities on the mean $x$ positions (not shown) yields a pattern speed of $60 \pm 14 \mathrm{~km} \mathrm{~s}^{-1} \mathrm{kpc}^{-1}$. Combining 

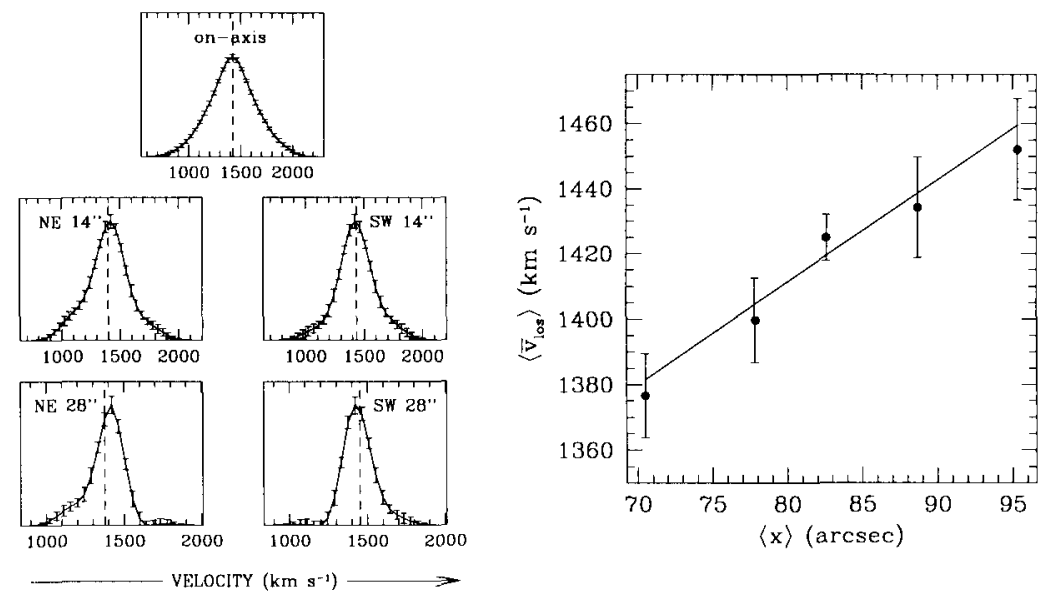

Figure 2. Left: Radial velocity distributions for the summed spectra of the five slit positions. Dashed lines indicate the mean velocities for the five slit positions. Right: Regression analysis of the mean velocities on the mean $x$-positions. The slope gives $\Omega_{p} \sin i$. Error bars are obtained by propagating photon errors through the analysis.

the pattern speed we found with the rotation curve of Kormendy (1984) yields a corotation radius at $69 \pm 15$ arcsec, which implies that the bar ends at a point close to, but within, the corotation radius.

\section{Conclusions}

This analysis has shown that, though still a little delicate, the TW method can be applied to (some) real galaxies and give plausible results. Good results can be obtained by co-adding data along an entire long slit and allowing for non-Gaussian line profile shapes: propagating the photon errors through this procedure gives results consistent with the scatter in the $\Omega_{p}$ values derived from different slit positions.

Though NGC 936 remains exceptionally regular among barred galaxies, it is at least reassuring that the measured pattern speed implies that the bar ends just inside the corotation radius.

\section{References}

Kent, S.M. 1987, AJ, 93, 1062

Kormendy, J. 1984, ApJ, 286, 132

Merrifield, M. R. \& Kuijken, K. 1995, MNRAS, 274, 933

Tremaine, S. D. \& Weinberg, M. D. 1984, ApJ, 282, L.5 